TITRE: NAVIGATEURS, MILIEU DE LA FINANCE ET BÉNÉVOLAT : SOUVENIRS DE TERRAIN ET RÉFLEXIONS TRANSVERSALES SUR LE RECRUTEMENT

Auteur(s): BÉATRICE EYSERMANN

Publication: ReCRUTEMENT ET CONSENTEMENT À LA RECHERCHE : RÉALITÉS ET DÉFIS ÉTHIQUES

PAGES: $161-176$

ISBN: 978-2-7622-0357-8

Directeurs: Ana MARIN, BÉATRICE EySERMANn ET MiChel T. GIROUX

URI: HTTP://HDL.HANDLE.NET/11143/14116

DOI: HTTPS://DOI.ORG/10.17118/11143/14116 


\section{Navigateurs, milieu de la finance et bénévolat : souvenirs de terrain et réflexions transversales sur le recrutement}

Béatrice Eysermann, Ph. D., coordonnatrice du Comité d'éthique de la recherche sectoriel en santé des populations et première ligne, CIUSSS de la Capitale-Nationale.

Résumé : Ce texte propose une lecture transversale de trois terrains de recherche menés entre 1996 et 2003, en France et au Japon. Souhaitant travailler sur le temps social dominant, l'auteure a exploré trois contextes particuliers visant le temps vécu hors du temps social (navigateurs solitaires, France), le temps capitaliste (à travers celui des grands financiers, Japon), et le temps gratuit dans une société capitaliste (le bénévolat, France). Trois terrains et trois expériences de recrutement totalement différentes, qui ont posé des questions cruciales: Comment rencontrer des navigateurs solitaires? Comment contacter les grands financiers? Comment recruter lorsqu'on endosse un double rôle de bénévole et de chercheuse sur le terrain? La démarche intuitive de l'auteure sera en outre réfléchie sous le prisme de son expérience québécoise en éthique de la recherche.

Mots-clés : Temps, recrutement, éthique, anthropologie. 


\section{Introduction}

Cet article fait le bilan des trois terrains de recherche que j'ai menés dans le cadre de mon cursus universitaire en France, entre 1996 et 2003, auprès de navigateurs solitaires, de grands financiers et de bénévoles. Le recrutement de participants est la clé de tout projet de recherche, et l'idée de ce colloque m'a poussée à faire une lecture transversale de ces terrains sous l'angle du recrutement, alors exempt de toute forme normative d'éthique de la recherche, puisque les recherches en sciences sociales en France ne sont pas sujettes au passage devant un comité d'éthique pour approbation¹.

Il est important de souligner d'entrée de jeu que la méthodologie en matière d'ethnographie en France teinte le terrain et son approche de manière significative. Ainsi, telle qu'elle me fut enseignée, la recherche ethnographique se fait d'abord et avant tout sur le terrain, c'est-à-dire en faisant de l'observation participante, en droite ligne avec l'héritage de Malinowski et de ses Argonautes du Pacifique occidental (1922). Le principe est alors simple : plonger dans son terrain le plus vite possible sans s'être acculturé soi-même par le biais de lectures, d'hypothèses ou d'un cadre théorique, et cela dans le but de tout voir avec le moins de filtres possible. C'est ce que rappelle Althabe (1990), pour qui l'ethnographie se fonde sur ce qu'il appelle la «communication ordinaire» entre le chercheur et ses «sujets» plutôt que sur des règles méthodologiques strictes encadrant la collecte de données sur le terrain. C'est ce que rappellent également Auger et Colleyn (2004: 79) lorsqu'ils écrivent que «l'efficacité de l'enquête de terrain réside sans doute moins dans la recherche consciente et active que dans un apprentissage spontané». Ils ajoutent: «L'art du terrain, comme il a parfois été appelé, ne s'apprend pas dans les livres» (ibid.).

Ne pourrait-on pas déduire de ces quelques lignes que le terrain relèverait davantage d'un savoir-être et d'un savoir-faire modulables plutôt que de règles méthodologiques à appliquer? C'est ce que révèle la lecture transversale de mes trois terrains de recherche, dont le recrutement oscille entre adaptation, ajustement, réajustement, débrouillardise et parfois audace, afin de parvenir à recueillir le nectar de notre matière première : les données brutes.

1. Toutefois, les débats en la matière ressurgissent régulièrement. Voir par exemple en 2008, la revue Ethnographique.org pour son numéro L'éthique en anthropologie de la santé : conflits, pratiques, valeur heuristique (numéro 17, 2008), ou en 2014, la revue du Journal des anthropologues, numéro 136-137, Désirs d'éthique. Besoins de normes? 


\section{Une problématique générale, trois recherches}

J'avais, au moment de ma première recherche (1996), une fascination pour le thème du temps. Une fascination insaisissable, avec un besoin irrépressible de comprendre «le temps», ce métronome implacable qui rythme nos vies tout en étant une entité insaisissable et intangible, puisque le temps est une abstraction².

Une abstraction qui peut toutefois se saisir par la façon dont les individus la vivent et l'incarnent. C'est ainsi que j'orientai ma problématique vers le temps social dominant ${ }^{3}$, qu'incarne l'horloge à deux puis trois aiguilles, surtout depuis la première révolution industrielle qui l'a fait évoluer vers le temps capitaliste de l'accumulation ${ }^{4}$ (Weber, 1994).

J'ai alors imaginé un triptyque qui devait correspondre à deux mémoires (une maîtrise et un DEA5) et une thèse de doctorat pour comprendre le temps social dominant - capitaliste - de la société dans laquelle je vivais. J'ai choisi trois groupes d'individus qui reflétaient, selon moi, trois déclinaisons de ce temps dans trois manières d'y vivre, soit en tentant de s'en soustraire (navigateurs solitaires), soit en l'incarnant (grands financiers), soit en le contournant (bénévoles d'actions caritatives).

Plongeons maintenant au cœur des terrains et du recrutement des participants.

\section{Navigateurs solitaires (1996)}

Mon intérêt pour les marins est né de ce que ma famille et moi avions vécu comme étant les affres des familles de marin, ma sœur ayant été embauchée du jour au lendemain en Martinique sur des voiliers dits de «charter $^{6} \%$. C'est parce que je voulais comprendre cette vie particulière que, plusieurs années plus tard, ce sujet de recherche s'est imposé tout naturellement à moi lors du choix d'un sujet pour la maîtrise. Je cherchais alors à répondre à la question suivante : Pourquoi les marins partent sur l'eau, et pourquoi ils y retournent? (Eysermann, 1997)

2. Alain Leroi-Gourhan (1967) y consacrera un chapitre dans son ouvrage Le geste et la parole (vol. 2). Pour lui, le temps fait partie des symboles de la société, au même titre que l'espace, les attitudes et le langage, ou l'esthétisme.

3. Parmi les caractéristiques de ce temps social dominant, on trouve celle de régulateur d'ordre social, d'horloge sociale, de donneuse de temps (Sue, 1995 ; Rifkin, 1996), de métronome pour l'organisation des rythmes collectifs (Sue, 1995) et pour le changement de rythme social (Nowotny, 1994). Dans ma thèse de doctorat, j'écrivais : "parce qu'il est partagé et utilisé par tous les membres de la société, il est le temps social dominant» (Eysermann, 2011 : 25).

4. Voir toute la démonstration dans la thèse, chapitre I: «Redéfinition du bénévolat dans le champ conceptuel de la linéarisation du temps, ou comment définir le temps «libre»» (Eysermann, 2011).

5. Diplôme d'études approfondies (DEA), équivalent à la première année du $3^{e}$ cycle et qui se solde par l'écriture d'un mémoire de recherche. Ce diplôme a été remplacé par le master à finalité de recherche en 2005.

6. Voiliers appartenant à des propriétaires qui engagent des équipages afin de pouvoir voyager avec leur famille ou leurs amis. Ces voiliers, avec équipage, peuvent aussi être loués à des touristes. 


\section{La préparation du terrain}

Pour préparer le terrain, j'ai lu les livres de marins bien connus comme Slocum (1990) ou Moitessier (1986), et j'étais totalement fascinée par ce que je découvrais en termes de voyage et de liberté. La version rédigée de leurs journaux de bord donne la sensation de voyager avec eux. Le profil des participants s'est donc d'abord défini à travers mes lectures : je souhaitais trouver ces navigateurs solitaires «à la Moitessier», marginaux de la société, vivant sur la mer et les océans.

J'ai aussi rassemblé les souvenirs de tout ce que ma sœur m'avait raconté sur la vie de marin pour parfaire ma préparation. Je savais ainsi que beaucoup vivaient en Bretagne, notamment un de ses amis, Pilou, avec qui elle avait traversé l'Atlantique. Mon but était de rejoindre le village de Bretagne où je trouverais cet homme et de côtoyer le plus de marins possible dans leur milieu. Je savais aussi que les marins étaient très hospitaliers, je pensais donc n'avoir aucun mal à trouver asile sur leur bateau pendant mon séjour. J'avais aussi arboré ce que je pensais être une tenue de circonstance - une veste de quart ${ }^{7}$ et un jean noir — dans l'idée de susciter, bien humblement, une reconnaissance identitaire visuelle chez les marins. J'étais prête. Je pris alors un train de nuit pour La Trinité-sur-Mer, où j'arrivai aux premières heures du matin. Nous étions en mars. J'avais deux semaines devant moi pour faire mon terrain.

\section{Premiers pas sur le terrain}

Dès mon arrivée, je confiai ma valise à un pêcheur du marché aux poissons. J'avais jusqu'à midi pour trouver asile sur un bateau et commencer mon terrain. Une fois les mains libres, je fis un tour dans les deux magasins d’accastillage 8 que j'avais repérés, pensant alors y trouver le profil de marins qui m’intéressait.

Après avoir écouté ma brève présentation, le vendeur du premier magasin me dit ne connaitre que des marins qui faisaient de la course en solitaire, et pas ces marins «à la Moitessier» que je recherchais. Un peu déçue, je me dirigeai vers le second magasin où je trouvai cette fois le type de marin souhaité : un homme qui préparait son bateau pour la traversée de l'Atlantique en solitaire. Ce premier informateur m'offrit l'hospitalité pour deux nuits sur son bateau, et je pouvais revenir lui confier ma valise pour le reste de la journée.

Comme je n'avais connu que des marins qui fumaient, le bar-tabac du village situé juste en face des quais me semblait être une place de choix pour établir mon «quartier général», y voir les allées et venues, faire la connaissance des marins et assurer une présence continue sur les lieux. Je décidai que j’y viendrais pour rédiger mon carnet de terrain et relire mes notes. C'est comme cela que, le soir de mon arrivée, mon premier informateur m’a présentée, moi et ma recherche, à des membres d'équipage de voilier qui repartaient dès le lendemain sur leur bateau. L'un d'eux me prêta son studio pour les deux semaines suivantes, en plus d'un vélo. J'étais prête à faire mon terrain.

\footnotetext{
7. Un «quart» est une période de temps pendant laquelle un marin est à la barre de son bateau. On appelle «veste de quart» le vêtement caractéristique que portent les marins en mer et qui comporte notamment des anneaux en métal pour pouvoir s'accrocher au bateau.
}

8. Magasin spécialisé dans la vente de matériel pour les bateaux. 
En parlant à ceux que je côtoyais jour après jour, il devenait alors évident que trouver tout un corpus de marins «à la Moitessier» serait impossible dans ce village : la voile étant un sport très onéreux, les marins doivent trouver des commanditaires et donc faire des courses pour publiciser leurs noms. Ce fait apparaissait d'autant plus évident que - et je le savais - les bateaux de course portent justement le nom de leur commanditaire? De fait, le profil de mes participants changea : je cherchais désormais des marins ayant déjà fait des courses à la voile en solitaire avec le soutien financier d'un ou de plusieurs commanditaires.

Je retournai donc au premier magasin d'accastillage pour obtenir le nom de quelques-uns. J'avais par ailleurs de la chance : une transat ${ }^{10}$ allait avoir lieu trois semaines plus tard et plusieurs marins se trouvaient déjà sur place avec leur maxi, des bateaux de 25 mètres. II semblait alors que j'arriverais plus facilement à trouver mon corpus.

II me fallait aussi partir en quête de ce Pilou que ma sœur avait connu. De fil en aiguille, je parvins à trouver une piste: on m'indiqua une maison dans laquelle se trouvait quelqu'un qui pourrait me renseigner. Une fois rendue, je me suis présentée en mentionnant l'objet de ma visite. Posté sur le seuil de la porte, l'homme me questionnait, relançait mes réponses, revenait sur plusieurs, et je sentais que tout pouvait basculer d'un moment à l'autre : j'avais en effet la certitude que, dans ce village où tout le monde se connaît, le moindre impair ou la moindre impatience de ma part pouvait me coûter la suite de mon terrain... C'est alors au bout de dix longues minutes que l'homme me tendit finalement la main pour me dire: «C'est moi, Pilou». Et c'est avec lui, et avec une célébrité de la voile des années 1980, que j'ai fait non seulement des entrevues, mais aussi, et de manière aussi impromptue qu'inespérée, un tour en bateau de quelques heures dans le froid mordant du petit vent de nord-est bien connu des Bretons.

Au final, ma présence continue sur le terrain a facilité mon intégration auprès d'un milieu qui semblait m'avoir adoptée. Tout le monde a fini par savoir qui j'étais sans m'avoir pour autant rencontrée, confirmant alors mon sentiment d'être observée. Ainsi, il m’est arrivé plusieurs fois d'être interpellée dans la rue par ces mots «Hé l'étudiante, si tu veux, je suis libre pour te parler», tandis que je ne restais jamais seule plus de dix minutes dans le bar-tabac. En effet, il y avait toujours quelqu'un qui venait s'asseoir à ma table pour me raconter la vie de marin, soit parce qu'il en était un, soit parce qu'il en connaissait un. J'ai même gardé le souvenir d'une femme, accotée au comptoir, qui m’a interpellée alors que je me rendais à la salle de bain. Elle m'a tout raconté de ce qu'elle vivait dans le monde de ces hommes de la mer, mais je n'avais ni crayon, ni papier, ni magnétophone pour l'enregistrer. Au terme de notre heure de discussion, je me précipitai sur mon carnet de terrain pour noter tout ce dont je me rappelais. Contre toute attente, cette entrevue informelle a beaucoup servi à l'analyse.

9. Par exemple, en 2017 : le trimaran Macif (compagnie d'assurance française) sur lequel François Gabart établit un nouveau record de tour du monde en solitaire ; le trimaran Banque Populaire sur lequel Francis Joyon et son équipage remportent le Record du Trophée Jules Verne; Peter Burling remporte la coupe de l'America avec son équipage sur le trimaran Emirates Team New Zealand (pour Fly Emirates).

10. Abréviation de «transatlantique»: course de bateau qui vise à traverser l'Atlantique, en solitaire ou en équipage. Chaque course a un nom spécifique. Il s’agit ici de la course Spi-Ouest France. 
La clé du recrutement a été le contexte dans lequel il prenait corps. Et il est un fait indéniable : plus je m'imprégnais du terrain, plus je saisissais les systèmes de représentation jusqu'à les vivre intérieurement. Ainsi, au fil des jours, je commençais à ressentir cet insondable fossé entre «marins» et «terriens» dont tous me parlaient; je me souviens même de m'être sentie prisonnière de la terre et d'avoir eu une furieuse envie de naviguer. Peutêtre l'aurais-je fait si je les avais côtoyés plus longtemps?

Je découvrais alors que comprendre l'univers des marins c'était aussi une manière de l'intégrer et, in fine, la passerelle la plus directe, pour moi la plus naturelle, vers le recrutement des participants. C'est comme cela qu'en deux semaines, outre mes carnets de terrain, j'ai effectué 17 entrevues formelles, plusieurs informelles, et côtoyé tous les profils de marins en présence : marins pêcheurs, marins en équipage, marins de course et un marin solitaire hors course. Ma stratégie de recrutement se dessinait clairement vers l'imprégnation du terrain, et vers la confiance que je saurais inspirer.

C'était mon premier terrain. C'est celui qui m’a le plus appris. C'est celui que j'ai le plus aimé.

\section{Grands financiers (1997)}

Un concours de circonstances a fait que je me trouvais au Japon lorsquej'ai fait ma recherche pour mon second mémoire ${ }^{11}$ en France. Si le temps des navigateurs solitaires en dehors de la société était celui que je nommais «le temps des choses et des éléments», j'entrepris de comprendre le temps capitaliste à travers ceux qui l'incarnaient dans les salles de marché ou trading floor. Comme je l'écrivais en introduction de mon mémoire, «je voulais me plonger dans un monde où la culture du temps était à ce point fondamentale qu'elle en devenait aliénante» (Eysermann, 1998: 3).

\section{Préparation du terrain}

Pour préparer le terrain, j'ai lu quelques livres sur les financiers pour me familiariser avec leur vocabulaire et repris l'étude de l'anglais, sachant que je devrais faire des entrevues en français et en anglais. J'ai également regardé une bonne dizaine de fois le film Wall Street d'Oliver Stone (1987) pour m'imprégner de l'atmosphère du monde de la finance et pour pratiquer mon anglais. Ma préparation dura environ deux mois. Je voulais travailler sur le stock exchange, c.-à-d. la bourse, qui, pour moi, «se jouait telle une pièce de théâtre dont les acteurs ne pensaient qu'à leur montre» (Eysermann, 1998: 3).

11. Voir note 5. 
Je partis ainsi un après-midi entier à la Bourse de Tokyo, la Tokyo Stock Exchange (TSE), pour trouver deux éléments qui m’ont forcée tout de suite à changer de lieu de recrutement. Le premier élément, pourtant prévisible, était de n'y voir que des travailleurs japonais. Le second élément, inattendu, était le suivant : je n'étais pas face à des acteurs se bousculant dans tous les sens pour réaliser des transactions d'achat et de vente, mais face à des hommes, apparemment paisibles, travaillant pour une bourse totalement électronique, et où il ne régnait pas, à ma grande déception, ni confusion, ni bruits, ni émulations. Et à 15 heures, tout s'est arrêté, c'était la fin des heures de marché au pays. Je ne trouverais pas là les informateurs dont j'avais besoin.

Ce n'est alors pas tant le profil des participants que le lieu d'exercice de leur profession qui changea. Les quelques banquiers avec qui j'avais discuté m'avaient parlé de deux catégories de travailleurs: les traders donnant les ordres d'achats et de ventes, et les brokers qui les exécutent. Mon observation de la TSE m'avait dissuadée de poursuivre dans cette voie, et j'optai alors pour les salles de marché des banques internationales pour y trouver des traders et des brokers francophones ou anglophones, faisant ou ayant fait du trading ou du brokerage, tout type de marchés confondus ${ }^{12}$.

Mais déterminer son terrain de recherche ne signifie pas que celui-ci est facile d'accès. C'est en me rendant au siège social d'une de ces banques internationales que je me suis retrouvée devant une véritable forteresse aux portes vitrées dont l'accès était de toute évidence réservé aux seuls privilégiés qui en possédaient le code. Inutile d'envisager de taper pour se faire ouvrir; il fallait trouver une stratégie pour rendre légitime ma présence sur les lieux.

\section{Élaborer une stratégie de recrutement adaptée au contexte}

En réfléchissant au milieu de la finance et à ses contraintes en termes de temps, je décidai d'une approche par contact direct et à distance. Pour cela, je choisis des noms au hasard dans l'annuaire de la Chambre de commerce française de Tokyo ${ }^{13}$, et rédigeai une lettre de présentation en français et en anglais que j'envoyai par télécopie. Cette lettre contenait les grandes lignes de mon projet, la promesse de confidentialité et une demande de participation à une entrevue pour m'aider dans ma recherche. J'y précisais que je les appellerais dans les deux jours pour sonder leur intérêt et leur laissais mes coordonnées téléphoniques.

L'idée d'une lettre de présentation envoyée par télécopie avait plusieurs atouts. J'ai vite compris que les moyens de communication rapides étaient la manière la plus sûre de capter leur attention et que, parmi ceux existant à cette époque, le fax était sans doute le meilleur. Cela permettait aussi de contourner plusieurs obstacles importants: se soustraire à une présentation trop longue par téléphone, et de la rallonger dans un anglais approximatif, leur faire perdre du temps pendant les heures de marché - ce qui m’aurait assuré un refus irréversible voire une fermeture du terrain. Sans compter que - je l'appris plus tard - le téléphone était l'outil de sollicitation continuel auquel les grands financiers étaient exposés, ce qui ajoutait à la pertinence de ne l'utiliser qu'à leur demande.

En plus des noms pris dans l'annuaire de la Chambre de commerce, je demandai à chaque participant de me donner le nom d'un ou deux grands financiers, et je procédais toujours par fax.

12. Exemples de types de marché : actions, obligations, devises étrangères, etc.

13. Qui venait de l'Ambassade de France et référençait les entreprises françaises de la capitale. 
Cette stratégie a eu 99 \% de succès; une seule personne a refusé. Un financier m’avait même téléphoné en me disant: «Bon, j'ai votre fax sur mon bureau depuis trois jours. Alors, vous voulez m'interroger ou pas?» J'ai évidemment pris rendez-vous avec cette personne, même si mon agenda était plein, car les plages de temps pour une entrevue devaient se situer en dehors des heures de marché14 et en dehors des heures de travail (qui dépendaient en outre des résultats des marchés). Trois plages étaient plus favorables : entre 11 heures et midi, après 17 heures et après 19 heures.

Mais avoir un rendez-vous ne signifiait pas pour autant que le recrutement était effectif. Les rendez-vous étaient parfois reportés jusqu'à quatre ou même cinq fois en raison d'impératifs dus aux fluctuations du marché, de rendez-vous de dernière minute avec des clients ou d'autres empêchements. Je devais ainsi confirmer chaque rendez-vous deux jours avant, le matin, et une heure avant, et commençais toujours la conversation, qui durait tout au plus quelques secondes, en demandant si c'était le bon moment pour téléphoner.

\section{Observations non préméditées}

Mon terrain m'a en outre permis de m'imprégner plus directement de l'atmosphère du monde de la finance par d'autres éléments imprévus. J'ai eu ainsi le privilège, par un de mes informateurs, d'aller observer une salle de marché de brokers pendant un après-midi entier. Les autres personnes présentes ignoraient totalement qui j'étais et la raison de ma présence. Une ambiance lourde pesait dans la salle. Dans l'introduction de mon mémoire, j'ai écrit:

Un des brokers a pris de vitesse un de ses collègues. Après quelques éclats de voix sans conséquence, chacune des deux parties s'est contenue et n'a plus rien dit, laissant place au calme de l'effusion des marchés, qui ne cessa jamais, sous le regard attentif de leur manager (1998: 7).

Cet extrait est révélateur de toute une stratégie interne orientée vers la division pour le profit que j'analysais en détail dans le corps de mon mémoire. Mais, pour l'heure, j’en étais repartie avec un terrible mal de tête.

J'ai eu l'opportunité également d'observer de près la relation de pouvoir très marquée entre trader et broker en étant conviée par un trader à rencontrer «son» broker dans l'un des restaurants les plus huppés de la ville. Selon ses termes, il fallait que je voie comment son broker allait «s'écraser devant lui», en ajoutant: «ll faut que vous voyez ça». Ainsi, sans qu'il n'ait jamais été informé de ma venue, et sans y avoir jamais consenti, ce broker est devenu mon hôte et paya la totalité de la facture. Il va sans dire qu'il a eu par la suite l'obligation d'accepter une entrevue - qui n'a duré ensuite que les quelques minutes qu'il lui a fallu pour se défaire de cette obligation, ce que je n'ai jamais dit au trader qui s'était bien sûr enquis du déroulement de ladite entrevue.

14. Dépendamment des marchés que les financiers suivaient. Rares étaient ceux qui se limitaient aux marchés de l'Asie. Beaucoup travaillaient aussi sur les marchés européens et américains, ce qui signifie qu'en tenant compte du décalage horaire ils pouvaient travailler jusqu'à tard le soir ou même la nuit. 
La stratégie utilisée pour approcher mes informateurs est empreinte de la compréhension d'un contexte de travail particulier dans lequel l'ethnographie par le porte-à-porte n'aurait rien donné. Il fallait s'ajuster. Par ailleurs, la confidentialité nécessaire et promise à mes informateurs exigeait que personne dans leur salle de marché ne soit au courant qu’ils allaient «parler». Et ce, pour deux raisons. La première est liée bien sûr à l'enquête de terrain elle-même. Mais la seconde illustre pleinement la réalité de ce terrain : à cet endroit, plus qu'ailleurs peut-être, l'information, c'est de l'argent. Ainsi, la moindre fuite produit un impact immédiat sur les marchés, sans parler d'un potentiel délit d'initié.

Au fil de mes quatre mois d'entrevues et d'observations, j'étais donc susceptible de recueillir des informations secrètes, et, si la confidentialité était toujours de mise, c'est sur le capital confiance qu'il leur fallait miser à mon endroit. Comme précédemment, c'est lors des entrevues que je tissais le lien de confiance, en démontrant notamment une compréhension exempte de tout jugement de leur univers. Si le travail de l'anthropologue n'est pas de juger, il peut parfois être confronté à des valeurs qui ne sont pas les siennes, voire en être dérouté. Et quoique ce fût mon cas, jamais je ne l'ai laissé paraître; j'interprétais toujours en fonction du contexte et de leur univers, renforçant ainsi le lien de confiance qui prêtait souvent à confidences. C'est ce lien qui m'a ensuite permis d'être invitée à faire des observations en terrain privé (repas au restaurant) ou, même, interdit (salle de marché), et d'obtenir d'autres noms.

\section{Bénévoles (1999-2002)}

Mes deux premiers terrains m'avaient permis de comprendre le temps dans deux univers opposés : le temps présent des choses et des éléments des marins solitaires devenait celui de l'instantanéité et de la simultanéité chez les grands financiers. Il me restait à comprendre le temps qu'on donne (gratuitement) dans un univers où on le vend, et c'est tout naturellement que je m'orientai cette fois vers les bénévoles. Mais lesquels choisir?

Je décidai de faire ma recherche à Marseille, en France, et commençai par chercher une association où faire mon terrain. Le travail commença par l'épluchage du registre des quelque 800 associations marseillaises, toutes vocations confondues. J'éliminai les associations pour les animaux maltraités (sensibilité personnelle), de même que les sports et loisirs, pour me concentrer sur les associations à vocation humanitaire, instaurées sur des bases laïques ou religieuses. À cela s'ajoute une typologie particulière à la France : les associations constituées en vertu de la loi $1901^{15}$, dont la légitimité est reconnue; et celles qui ne le sont pas et donc risquaient de disparaître durant ma recherche.

Ma première intention était de comparer les deux associations dites humanitaires que j’avais sélectionnées : une, militante, à vocation de faire sortir les gens de la misère par la culture et l'éducation; et l'autre, qui s'inscrit dans la tradition de la charité et du don. Après un essai dans chacune de ces associations, je décidai de ne me concentrer que sur celle fondée sur la tradition chrétienne de charité.

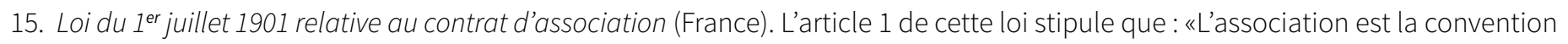
par laquelle deux ou plusieurs personnes mettent en commun, d'une façon permanente, leurs connaissances ou leur activité dans un but autre que de partager des bénéfices. Elle est régie, quant à sa validité, par les principes généraux du droit applicables aux contrats et obligations.» Les associations constituées en vertu de cette loi ont un statut pérenne. Pour plus d'informations, voir le lien suivant: https://www.legifrance.gouv.fr/affichTexte.do?cidTexte=LEGITEXT000006069570 


\section{Commencer par de l'observation participante}

Lors de ma première visite à l'association, après avoir expliqué mon vaste objet d'étude, «le bénévolat», la secrétaire m'a orientée vers l'accueil de nuit : «ll y a une activité avec les sans-abri ce soir. Si vous voulez, venez.» J'ai immédiatement accepté, démontrant par là même, pensais-je, mon intérêt et déjà, mon engagement. Dès le premier soir, je distribuais des soupes à des sans-abri, ce qui laissa en moi la trace indélébile de ce premier souvenir : un homme mangeant une boîte pour chats. Après m'être ressaisie (le sans-abri avait-il vraiment, en plus, besoin de mes larmes), cette première impression : approcher les sans-abri était d'une facilité déconcertante. L'observation participante commençait.

Au fil des deux ans de terrain, et de ma présence constante à l'association, j'ai probablement vécu toute la palette des émotions possibles, que ce soit au contact des sans-abri ou avec les bénévoles qui parfois me testaient. J'ai participé à toutes les (très) nombreuses réunions organisées par l'association, assuré la coordination de l'activité de nuit pendant deux mois et participé à l'évaluation de cette activité pour un organisme externe.

\section{Recruter en terrain connu?}

Le recrutement des participants était loin d'aller de soi : tout le monde faisait fi de mon identité d'étudiante-chercheuse pour ne me considérer que comme simple bénévole. Je me suis alors inspirée de la stratégie de recrutement des grands financiers pour me distancer du terrain et revêtir le chapeau d'anthropologue - que je n'avais pour ma part jamais quitté.

Je rédigeai une lettre titrée «une anthropologue parmi vous» dans laquelle j'expliquais ma présence à l'association, mon terrain, et que j'allais procéder à l'étape des entrevues. J'y décrivais les critères de confidentialité et de liberté de participation, et leur demandais de me contacter. Les neuf salariés et quelques bénévoles ont accepté de m'accorder une entrevue, ce qui s'inscrivait tout à fait dans la vocation du rendre service associatif. Mais les choses avaient changé : tous me considéraient à présent à travers mon «désengagement» bénévole (Fillieule et Broca, 2001).

Je soulignerai ici un élément intéressant: à ma grande déception, les entrevues que je faisais ne m’apprenaient rien de plus que mes observations profanes. Souffrais-je d’acculturation? Les bénévoles démontraient quant à eux un humanisme et un altruisme parfois en contradiction avec mes observations de terrain ${ }^{16}$. Ce n'est que plus tard, une fois sortie du terrain et en analysant les données, que je saisis toute l'ampleur et l'envergure du temps associatif, en apparence flottant et insaisissable, de même que le niveau d'acculturation qui fut le mien au bout de deux années consécutives.

Pour clore mon terrain, j'ai également voulu recruter des sans-abri, ce que je fis, par grand vent, un soir froid de janvier. Je me suis présentée comme une «petite étudiante» qui faisait un travail pour l'Université, et je posai mes questions aux trois sans-abri qui se tenaient là. L'intervenant qui m'accompagnait devait assurer une présence discrète et muette, mais il ne s'y tint pas et posa des questions pour son travail. Cela a quelque peu court-circuité mon entrevue informelle, mais cela fait aussi partie du terrain.

16. Les exemples de contradiction sont nombreux. Je me souviens notamment du discours humaniste très senti d'une bénévole envers les sans-abri, alors qu'avant de partir en tournée de nuit, cette dernière s'évertuait à cacher les denrées sous les sacs ou les containers car, disait-elle, ils allaient les voler. Chose que je n'ai pour ma part jamais observée. 
À la différence des deux premiers terrains, ma présence continue sur les lieux pendant une période de deux ans faisait de moi une bénévole comme les autres. Les acteurs en présence étaient d'ailleurs bien plus intéressés par mon engagement indéfectible que par le vrai but de ma présence, du moins dans les premiers temps. Au début, je me présentais toujours aux bénévoles et dans toutes les réunions comme une étudiante de doctorat qui faisait du bénévolat pour sa recherche. Cela ne semblait intéresser personne. Je le fis ensuite de façon plus sporadique, puis j'ai arrêté.

Le défi, plus tard, fut de changer d'identité à leurs yeux pour redevenir ce que je n'avais jamais cessé d'être: une doctorante sur son terrain de recherche. Si le lien de confiance m'a permis de recruter des participants sur les deux premiers terrains, rappeler aux bénévoles la (véritable) raison de ma présence a semblé parfois ternir ou émousser ce lien : j'étais parfois prise à partie et devais me repositionner souvent pour les convaincre de ma neutralité (Eysermann, 2010). J'avais compris qu'il y avait un moment clé à ne pas dépasser pour recruter et faire les entrevues, etj'ai respecté ce moment. L'étape suivante ne pouvait être que mon retrait de l'association, et nous le savions tous.

Quand j'y suis retournée en 2003 pour valider mon analyse, le temps avait fait son œuvre, et je fus accueillie comme une vieille amie «qui a fait sa thèse sur l'accueil de nuit». J'ai pu même bénéficier d'un passe-droit pour intégrer une équipe de tournée de nuit le soir même sans passer par le nouveau planning organisationnel qui avait été mis en place dans l'intervalle.

\section{Réflexions transversales sur le recrutement}

Mes trois terrains de recherche ont en commun la familiarisation des contextes par la lecture et tout autre élément dont je pouvais disposer. C'est ce qui me donna suffisamment confiance pour entrer en contact avec le terrain et savoir comment faire mon recrutement. Ce fut néanmoins très différent d'un terrain à l'autre.

Pour les marins, nous avions, mes parents et moi, toutes nos années d'expérience comme famille de marins, et ma sœur nous en avait présenté plusieurs. Je pensais en comprendre la mentalité, et je ne m'étais pas trompée. Le fait d'avoir une sœur qui a navigué dans ce milieu m'a ouvert aussi les portes de ce monde. J'ai approfondi cette connaissance profane et familiale par la lecture de magazines de voiles et de ces carnets de bord des grands marins solitaires. Ce qui m'a très vite permis de m'intégrer à leur monde et de collecter beaucoup de données pour mon analyse.

Pour ce qui est des financiers, ma brève préparation m’a permis de comprendre le contexte temporel dans lequel ils baignaient douze à quinze heures par jour, à savoir que le temps c'est de l'argent et qu'il faut faire de l'argent. En réponse, je n'avais d'autre choix que d'orchestrer le recrutement autour de l'injonction de ne surtout pas leur faire perdre de temps. Il fallait donc ruser et créer le moyen le plus astucieux de les motiver à participer à ma recherche. La clé du recrutement était là.

Je n'avais jamais fait de bénévolat humanitaire avant d'entrer dans l'association pour y faire mon terrain. L'observation participante a débuté dès le premier soir avec les bénévoles auprès des sans-abri, et ensuite quotidiennement dans la sphère associative où je passais mes journées. Ma présence continue pendant deux ans aura été autant un formidable vecteur pour collecter des données qu'elle n'est devenue un défi lorsqu'il fallait recruter en terrain connu. Car, et il faut le souligner, devenir bénévole pour ma thèse m'avait forgé, malgré moi, 
une double identité dont il me fut impossible de me départir sans conséquence : après les entrevues, j’ai bien senti que je n'étais plus vraiment la bienvenue. Une pause était nécessaire, et il fallait la respecter.

Ces contextes distincts mettent en relief l'aspect intuitif du terrain, tant il faut savoir sentir, jauger, juger de tous les éléments à disposition pour saisir le bon moment pour recruter. Et j’ai pu constater que c'est cette connaissance de leur milieu qui inspirait aussi confiance aux participants potentiels, qui réciproquement m'ont aidé à enrichir ma collecte de données : bien des marins se sont assis à ma table dans le bar-tabac du coin pour me parler de leur vie; j'ai fait de l'observation dans une salle de marchés (interdite) et dans un restaurant; les bénévoles, eux, me faisaient confiance à travers mon engagement sans limites, et m'appréciaient d'abord pour cet engagement.

Donc ma stratégie a été dans tous les cas d'inspirer confiance, d'en savoir assez pour que les personnes présentes me prennent au sérieux et de ne pas en savoir trop, ce qui risquerait de compromettre la raison d'être de ma présence; en effet, c'est à eux de m'aider à comprendre leur univers. Faire de l'observation participante m'a aussi permis d'avoir les mêmes référents que mes participants lors des entrevues en plus de pouvoir truffer mes mémoires ou ma thèse d'exemples vécus.

\section{Pour conclure : Passer outre un comité d'éthique de la recherche (CER)?}

Je suis tout naturellement obligée de terminer mon texte en parlant du comité d'éthique de la recherche, puisqu'aucune de ces recherches n'a fait l'objet d'une évaluation en ce sens.

En prenant appui sur mes neuf années passées à la coordination d'un CER à Québec, je ferai trois constats :

1. Jamais je n'aurais pu deviner le déroulement de mes terrains et moins encore le décrire dans un protocole bien ficelé pour qu'il soit évalué adéquatement. Les profils théoriques de marins et de grands financiers que je recherchais se sont révélés caducs dès mon arrivée sur le terrain. J'aurais dû faire des amendements en cours de route, ici depuis la Bretagne ou le Japon, et attendre le feu vert. Si les CER demandent beaucoup de précision dans les protocoles, peut-être que certaines circonstances exigent au contraire plus de souplesse eu égard aux imprévus de terrain et de ses acteurs.

2. Pour ce qui est du recrutement en passant par des tiers, comme les CER ont tendance à le prôner pour toutes les recherches, j'ai pu en bénéficier, mais pas de manière systématique, et seulement sur l'invitation expresse d'informateurs voulant m'aider. C'est ainsi que les marins ont parfois servi d'intermédiaires suivant leur propre initiative ou m'interpellaient directement dans la rue (supra). Pour les financiers, je présume que j'aurais écrit dans mon protocole que je passerais par les réceptionnistes, avec un échec assuré à la clé. Comment les aurais-je approchées puisque je ne pouvais pas entrer dans ces forteresses vitrées? Qui l'aurait fait pour moi? Il aurait fallu justifier de la pertinence d'un contact direct et à distance, qui relevait bien plus d'une compréhension intuitive du contexte que de données théoriques. 
3. Plusieurs données ont été collectées spontanément dans des contextes imprévus. Pour en rappeler quelques-uns : lors de la navigation avec deux marins, de l'observation d'une salle de marché et dans le restaurant; des différents rôles que j'ai endossés à l'association (bénévole, responsable de l'activité, évaluatrice externe), et de l'entrevue avec les sans-abri - qui a presque tourné en entrevue professionnelle pour l'intervenant qui m'accompagnait. À bien y réfléchir, aucun de ces exemples n'était prévisible : ils émanaient des participants eux-mêmes, qui attendaient mon acquiescement immédiat. C'est aussi là, entendons-nous, toute la beauté du terrain. Aurais-je profité d'autant de spontanéité si j'avais dû tout prévoir, ou tout faire approuver avant de donner une réponse? Si l'éthique de la recherche donne des balises solides, le chercheur pourrait aussi, dans de telles circonstances, jauger du niveau de risque, faire parler son expérience et prendre les bonnes décisions. Sur mes terrains, avec le recul, je pense pouvoir dire que c'est ce que je fis, et j'en savoure encore la richesse aujourd'hui.

Alors, pourrait-on se passer des CER? Non. En neuf ans dans un CER, j'ai plusieurs fois été soulagée que des protocoles soient ainsi évalués, car ils nécessitaient une sévère révision au plan éthique. Par contre, il ne faut pas penser ce processus en sens unique, ce qui est trop souvent le cas : les chercheurs doivent parler et faire valoir leur contexte de recherche puisque ce sont les seuls à le connaître vraiment. Les chercheurs, certains, font fausse route quand ils cherchent à répondre ce qu'ils supposent que le CER veut entendre au lieu de mordre dans leur connaissance du contexte. Ce qui donne d'ailleurs des mauvaises réponses, vagues, creuses. Et on les repère tout de suite. Je n'ai pas vu beaucoup de recherches pour lesquelles les chercheurs ou les étudiants ne connaissaient rien de leur terrain. J'ai même déjà vu les membres du CER changer leur position suite à l'argumentaire de chercheurs qui explicitaient les raisons pour lesquelles ils ne pouvaient pas souscrire à une de leurs recommandations.

Alors ainsi, pour les recherches en sciences sociales du moins, les chercheurs et les étudiants ont la responsabilité d'élaborer leur protocole au plus précis de leur connaissance de terrain afin de viser une saine et profitable collaboration plutôt que de construire - de part et d'autre, d'ailleurs - des lignes d'incompréhension. Pourquoi? Parce que le recrutement est, la plupart du temps, du moins pour les sciences sociales, le point phare de l'évaluation éthique des CER, avant même le consentement. Le dialogue ne peut alors que gagner à être éclairé et ouvert, encore une fois, de part et d'autre.

\section{Annexe : résultats de recherche ou l'immanence du temps présent}

Mes recherches m'ont appris que le temps social dominant se muait inévitablement vers une seule temporalité : le présent. C'est celui du temps des choses et des éléments chez les marins, qui ne revêt que $10 \%$ du temps de voyage, qui les pousse à retourner sans cesse sur les mers et les océans. Ce temps présent où ils se sentent en harmonie avec leur univers et libres de tout, de tous, où ils savourent un instant après l'autre.

Si les marins savourent ce temps qu'ils gagnent pendant leur voyage, les financiers sont prisonniers du temps capitaliste. Rivés sur le temps de l'accumulation pour le futur, ils sont pris au piège de la temporalité des marchés dont ils ne savent s'extraire : le temps présent de l'instantanéité ou de la simultanéité des transactions d'achats et de vente. Je n'ai rencontré que des millionnaires sur mon terrain. Ils m'ont tous dit que le premier million est le premier objectif, mais qu'il est très vite atteint. Ainsi, pour garder les pieds sur terre, ils ne pensent pas en termes de millions, mais d'unité : «On a un, on veut deux», etc. Dans cet univers capitaliste par excellence, ils se sentent contraints de travailler de 15 à 18 heures par jour; l'un d'eux m'a même dit qu'il aimerait ne jamais dormir. Serviteurs de ce temps de l'accumulation, se déclarant incapables de décrocher ou de prendre des vacances afin de pouvoir surveiller sans cesse les fluctuations des marchés et leurs positions, ces millionnaires vivent le plus absurde des paradoxes : ils n’ont pas le temps de dépenser leur argent... 
Quant aux bénévoles, je me suis posé la question du temps gratuit et de sa signification dans un univers où on le vend. À défaut d'accumuler des millions, ce sont les heures que les bénévoles accumulent, tant leur temps devient élastique pour les salariés de l'association qui l'étirent sans cesse pour en repousser les limites. Mais la surprise fut sans doute de constater que, réciproquement, les salariés sont d'insoupçonnés donneurs de temps. Les bénévoles ne se cachaient pas pour affubler les salariés du paradoxe selon lequel percevoir un salaire et avoir bon cœur sont incompatibles. De fait, les bénévoles faisaient des salariés de vrais prestataires dévoués exclusivement à leur service, et n'avaient de cesse d'user et d'abuser de ce privilège qu'ils se donnaient, les empêchant ainsi de faire le travail pour lequel ils étaient réellement payés. Tous les salariés se voyaient alors contraints de faire des heures supplémentaires, le soir ou les fins de semaine. Gratuitement cette fois. Les tensions entre bénévoles et salariés cohabitaient avec l'obligatoire bonne entente et légèreté qui régnaient toujours à l'association.

Ma conclusion fut celle-ci : le temps des bénévoles comme celui des salariés est élastique, et aucune des deux catégories d'acteurs n'a conscience du temps qui lui est donné. Ceci crée une incompréhension temporelle, source de tensions et de malentendus, qui ne se résolvent jamais. Car, si le temps gratuit est invisible aux yeux de celui qui le reçoit, le corollaire est d'être extensible à l'infini ou de ne jamais suffire. Et les temps s'étirent. Et la boucle recommence.

Trois contextes, trois temps présents, toujours accolés au temps social dominant auquel, quoi qu'on fasse, on ne peut se soustraire. J'ai voulu à travers ces recherches comprendre aussi bien le temps de ma société que ma fascination pour ce thème. Si j'ai l'impression d'avoir répondu à ma question, ma fascination reste entière. 


\section{Bibliographie}

Althabe, G. (1990). Ethnologie du contemporain et enquête de terrain, Terrain, 14, 126-131. DOI : 10.4000/terrain.2976

Augé M. et Colleyn J.-P. (2004). L'anthropologie, Chapitre III. Le terrain. Paris : PUF.

Eysermann B. (2011). L'ouvre des donneurs de temps dans notre société. Bénévoles et salariés de l'humanitaire dans une association caritative marseillaise, Éditions universitaires européennes.

Eysermann B. (2010). Un terrain dans une association caritative marseillaise (France) à l'épreuve de l'éthique de la recherche (Canada). Éthique, méthodologie et responsabilité, Éthique publique, 12 (1), 179-200. DOI: 10.4000/ethiquepublique.185

Eysermann B. (1998). Trader/broker versus capitalisme: vers un temps de la postmodernité? (Mémoire de DEA, Université d'Aix-Marseille I, France).

Eysermann B. (1997). Vivre le temps présent. La navigation à voiles en solitaires, (mémoire, Université d'Aix-Marseille I, France).

Fillieule, O. et Broqua C. (2001). Désengagement et conséquences biographiques de l'engagement. Dans Actions associatives, solidarités et territoires (p. 157-162). Saint-Étienne (France) : Presses universitaires de Saint-Étienne.

Leroi-Gourhan A. (1967) Le geste et la parole, Albin Michel, vol 2.

Malinowski. (1922), Les Argonautes du Pacifique occidental

Moitessier, B. (1986). La longue route, Arthaud.

Nowotny, H. (1994). Time. The Modern and Postmodern Experience, traduit de l'allemand par Neville Price, Polity Press.

Rifkin, J. (1996). La fin du travail, La découverte.

Slocum, J. (1990). Navigateur en solitaire, Babel-Actes Sud.

Sue, R. (1995). Entre travail et temps libre : l'émergence d'un secteur quaternaire. Cahiers internationaux de sociologie, 99, 401-415.

Weber, M. (1994). L'éthique protestante et l'esprit du capitalisme, Plon, Pocket.

\section{Numéros de revue}

L'éthique en anthropologie de la santé : conflits, pratiques, valeur heuristique, 17, 2008 : http://www.ethnographiques.org/Numero-17-novembre-2008

Désirs d'éthique. Besoins de normes?, 136-137, 2014: https://jda.revues.org/656 